\title{
Gero Erdmann-Preis für vergleichende Area-Forschung 2016
}

\section{Marianne Kneuer}

Online publiziert: 30. November 2016

(C) Springer Fachmedien Wiesbaden 2016

Liebe Kolleginnen, liebe Kollegen,

das ist eine Premiere, wir verleihen den Gero Erdmann-Preis für Vergleichende Area-Forschung heute erstmals. Daher möchte ich kurz etwas zur Genese des Preises sagen, bevor ich zu den Laudationes an die beiden Preisträger schreite sowie zur Verleihung der Urkunden.

Als unser Kollege Gero Erdmann viel zu früh im Sommer 2014 verstarb, hatten wir den Wunsch, einen Weg zu finden, sein Andenken zu bewahren. Wenn ich sage wir, dann meine ich damit den AK Demokratieforschung, dem Gero viele Jahre als Sprecher angehörte, sowie die ZfVP, dessen Mitgründungsherausgeber er war. Die Idee eines Preises wurde geboren. Und da Gero Erdmanns Forschungsfeld, das er als Wissenschaftler am GIGA Hamburg vertrat, die Vergleichende Area-Forschung war, stand das Thema schnell fest. Es handelt sich um einen Nachwuchspreis, der eine herausragende Dissertation auf diesem Gebiet auszeichnet, denn Gero lag der Nachwuchs besonders am Herzen. Der Preis wird alle zwei Jahre vergeben und ist mit $1500 €$ dotiert.

Zwar gab es keine Flut, aber doch so viele Einreichungen, dass wir einen kompetitiven Auswahlprozess durchführen konnten. Bedanken möchte ich mich auch bei meinen Jurykollegen, Hans-Joachim Lauth und Bert Hoffmann. Die Jury entschied konsensuell, dass sie sich bei zwei Arbeiten nicht für eine Reihung entscheiden konnte. Daher werden wir dieses Jahr zwei hervorragende Dissertationen auszeichnen: nämlich die von - alphabetisch - Dr. Johannes Gerschewski und Dr. Dorothea Keudel-Kaiser. An dieser Stelle möchte ich beide Preisträger nach vorne bitten.

Prof. Dr. M. Kneuer $(\varangle)$

Institut für Sozialwissenschaften, Universität Hildesheim, Universitätsplatz 1, 31141 Hildesheim, Deutschland

E-Mail: kneuer@uni-hildesheim.de 
Lassen Sie mich nun die beiden Arbeiten laudatieren:

Die Dissertation von Dr. Gerschweski „Stabilizing Autocratic Rule. Legitimation, Repression and Co-optation in East Asia's Autocracies“ ist im Bereich der Autokratieforschung angesiedelt. Auf der Basis eines theoretisch hergeleiteten Modells - des Drei-Säulen-Modells - untersucht die Arbeit 13 ostasiatische Länder.

Die Gutachter stellten fest, dass die Dissertation eine außergewöhnliche theoretische Durchdringung aufweist, ebenso wie einen überaus originellen konzeptionellen Zugang in Form des Drei-Säulen-Modells von Legitimation, Repression und Co-optation. Die empirische Analyse - eine fuzzy set QCA kombiniert mit der in-depthAnalyse Nordkoreas - ist sehr anspruchsvoll umgesetzt.

Im Namen der Jury gratuliere ich für diese Leistung.

[Überreichung Urkunde]

Die Dissertation von Frau Dr. Keudel-Kaiser „Government formation in Central and Eastern Europe. The case of minority governments“ bewegt sich im Bereich der Regierungsforschung. Das Erkenntnisinteresse zielt auf Faktoren, die die Bildung von Minderheitsregierungen determinieren. Die Arbeit analysiert 39 Fälle von Regierungsbildungen in acht ostmitteleuropäischen Ländern.

Die Gutachter halten fest, dass die Studie durch ihr konsequent und schlüssig umgesetztes methodisches Vorgehen besticht, das auf einer sehr guten theoretischen Grundlage beruht. Der Anspruch liegt dabei darauf zu zeigen, ob in der OECD-Welt gewonnene Erkenntnisse über die Bildung von Minderheitsregierungen reisen, sprich auf Ostmitteleuropa übertragbar sind. Mit dieser Arbeit wird eine Forschungslücke der Parteienforschung geschlossen.

Ich möchte auch Ihnen im Namen der Jury sehr herzlich gratulieren.

[Überreichung der Urkunde]

Beide Arbeiten, liebe Kolleginnen und Kollegen, bestechen in ihrer Theoriegeleitetheit sowie in ihrer originellen empirischen Aufarbeitung. Sie wurden beide auf Englisch verfasst, was den Internationalisierungsgrad der deutschen Nachwuchsforschung belegt. Zudem leisten aus Sicht der Jury beide Arbeiten wertvolle Erkenntnisse für die internationale Debatte in den beiden jeweils fokussierten Bereichen. Insofern freuen wir uns, dass mit diesem ersten Gero Erdmann-Preis zwei so bemerkenswerte Arbeiten ausgezeichnet werden konnten.

Von links: Johannes Gerschewski, Marianne Kneuer, Dorothea Keudel-Kaiser (Foto: Alexander Kobusch)

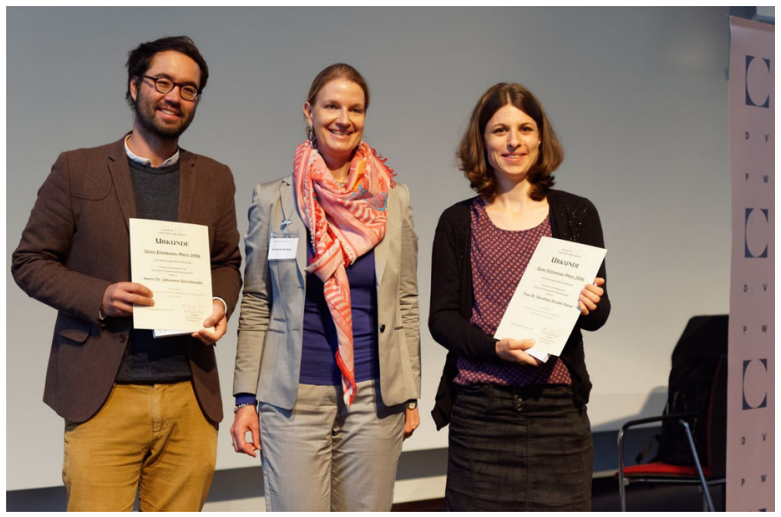

